\title{
Téoros
}

Revue de recherche en tourisme

\section{Attraits, attractions et produits touristiques}

Trois concepts distincts dans le contexte d'un développement

touristique régional

\section{François de Grandpré}

Volume 26, numéro 2, été 2007

L’attractivité touristique des territoires

URI : https://id.erudit.org/iderudit/1070941ar

DOI : https://doi.org/10.7202/1070941ar

Aller au sommaire du numéro

\section{Éditeur(s)}

Université du Québec à Montréal

\section{ISSN}

0712-8657 (imprimé)

1923-2705 (numérique)

Découvrir la revue

\section{Citer cet article}

de Grandpré, F. (2007). Attraits, attractions et produits touristiques : trois concepts distincts dans le contexte d'un développement touristique régional. Téoros, 26(2), 12-18. https://doi.org/10.7202/1070941ar 


\section{Attraits, attractions et produits touristiques}

\section{Trois concepts distincts dans le contexte d'un développement touristique régional}

\section{François de Grandpré}

En tourisme, les concepts de « produits » et d'«attractions » sont largement utilisés, tant dans la littérature scientifique que dans le langage courant et la publicité. Curieusement, ces concepts n'ont pas encore de signification unique, claire et reconnue. Quant à la notion d' " attraits ", elle ne semble pas avoir fait l'objet d'autant d'attention et est peu conceptualisée.

Ces concepts, en particulier celui de produit, sont importants en tourisme. C'est par exemple l'absence d'un mode de production traditionnel d'un produit homogène qui fait hésiter les économistes à qualifier le tourisme d' "industrie" (Smith, 1994). Cette activité économique compte pourtant parmi les plus importantes de la planète. La signification que nous donnons à ces concepts peut avoir des conséquences sur la façon dont nous percevons l'activité touristique et la façon dont nous mettons en valeur nos territoires. C'est de ce dernier point qu'il sera question dans la conclusion. Auparavant, examinons les écrits sur le sujet et comment ces concepts et ces notions peuvent s'articuler à l'intérieur d'une compréhension systémique du tourisme.

Tel qu'indiqué dans l'Encyclopédie du tourisme (Jafari, 2000), tout ce qui est acheté lors d'un voyage peut être appelé un produit touristique. Plus exactement «un produit est tout ce qui peut être offert pour satisfaire un besoin ou un désir » (Kotler et al., 2000 : 10). II y a des cas où le concept de produit s'applique aussi à des services gratuits, comme certains loisirs (exemple: une balade à vélo), ou à des biens publics (un paysage), donc où le produit ne nécessite pas une transaction explicite entre un

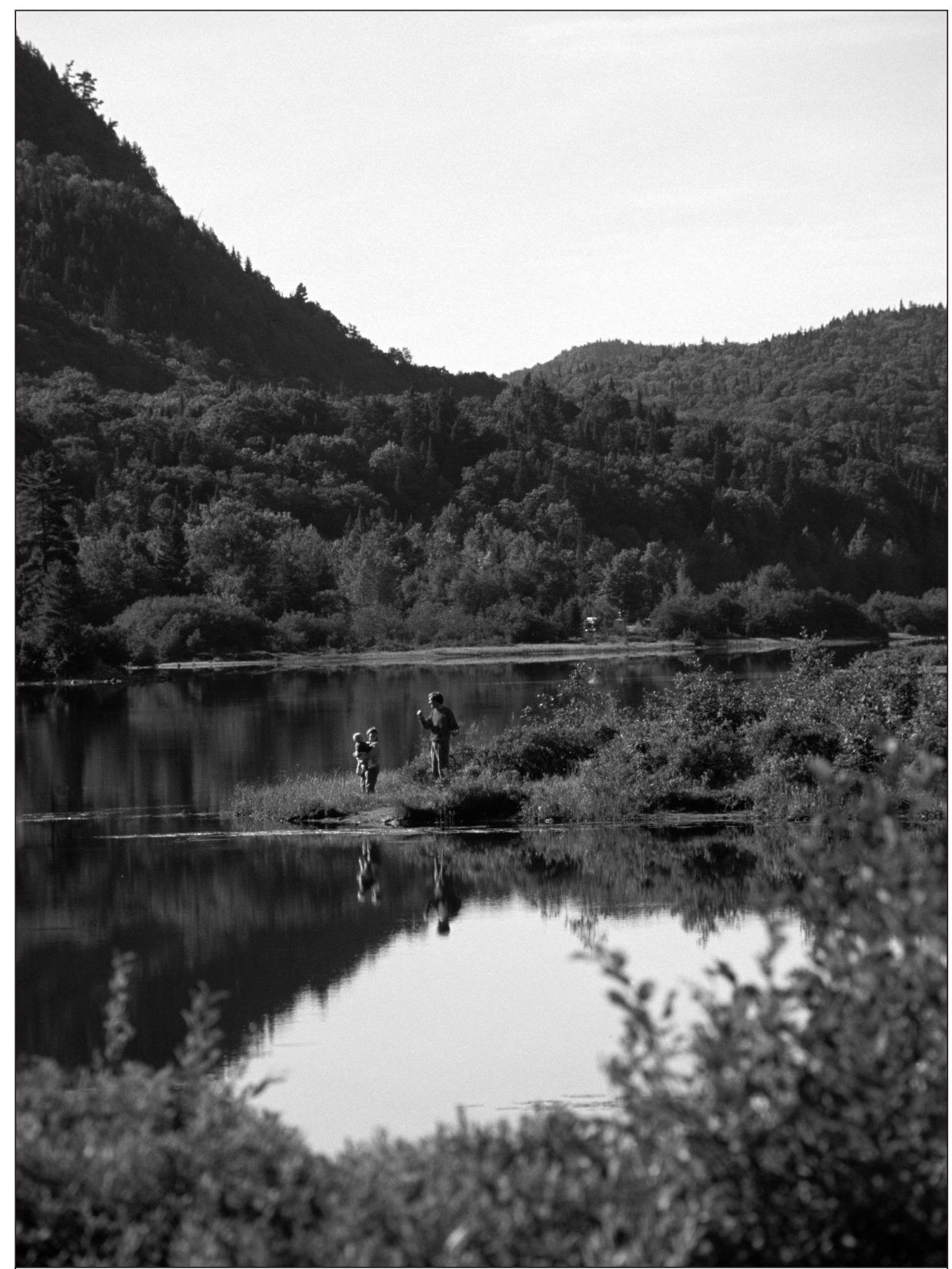

Laurentides, Parc national du Mont-Tremblant, Laurentides.

Photo : Marcel Gignac, Tourisme Québec. 
acheteur et un vendeur. Kotler va plus loin en affirmant qu'une idée peut être considérée comme un produit, au même titre qu'un bien ou un service. C'est cette flexibilité de la notion de produit qui nous a amené à nous demander si le produit touristique ne serait pas justement qu'une idée, un idéal à atteindre lors d'un séjour hors de son lieu habituel de vie, un état d'esprit. N'entendons-nous pas souvent dire que l'expérience touristique se passe avant tout dans la tête du touriste? Attardons-nous davantage à la notion de produit touristique et regardons quelle place occuperaient les notions d'attractions et d'attraits dans ce contexte.

\section{Le produit touristique}

Dans la littérature scientifique, le produit touristique prend une multitude de significations. En plus de présenter sa propre modélisation du produit touristique, Smith (1994) recense près d'une vingtaine d'auteurs (dont Medlik et Middleton, Pearce, Butler, Gunn, Jefferson et Lickorish, Sasser, Olson et Wyckoff) qui se sont appliqués à définir ce concept. Le produit rassemble une grande variété d'éléments. Aux dires de Medlik et Middleton, par exemple (rapportés dans Smith, 1994), le produit est un assortiment d'activités, de services et de bénéfices qui constitue l'expérience touristique dans sa globalité. Par ailleurs, c'est aussi l'offre à l'échelle d'une entreprise touristique.

II n'est pas surprenant alors de trouver une liste hétéroclite dans l'énumération des produits touristiques offerts sur un territoire. Ainsi, Smith (2003), dans une énumération des produits touristiques caractéristiques du Canada, identifie un grand nombre d'éléments qui vont des particularités de certaines nations (autochtones, québécoise, acadienne), en passant par des lieux, des équipements récréotouristiques, des événements, des structures d'accueil, des activités, des équipements culturels, et la liste continue.

II est assez largement reconnu aujourd'hui que le produit touristique peut être l'ensemble des activités réalisées par le touriste à partir du moment où celui-ci quitte son espace habituel de vie et ce, jusqu'à ce qu'il y retourne. Pour plusieurs, le produit touristique correspond ainsi à l'ensemble du périple. Les diverses consommations

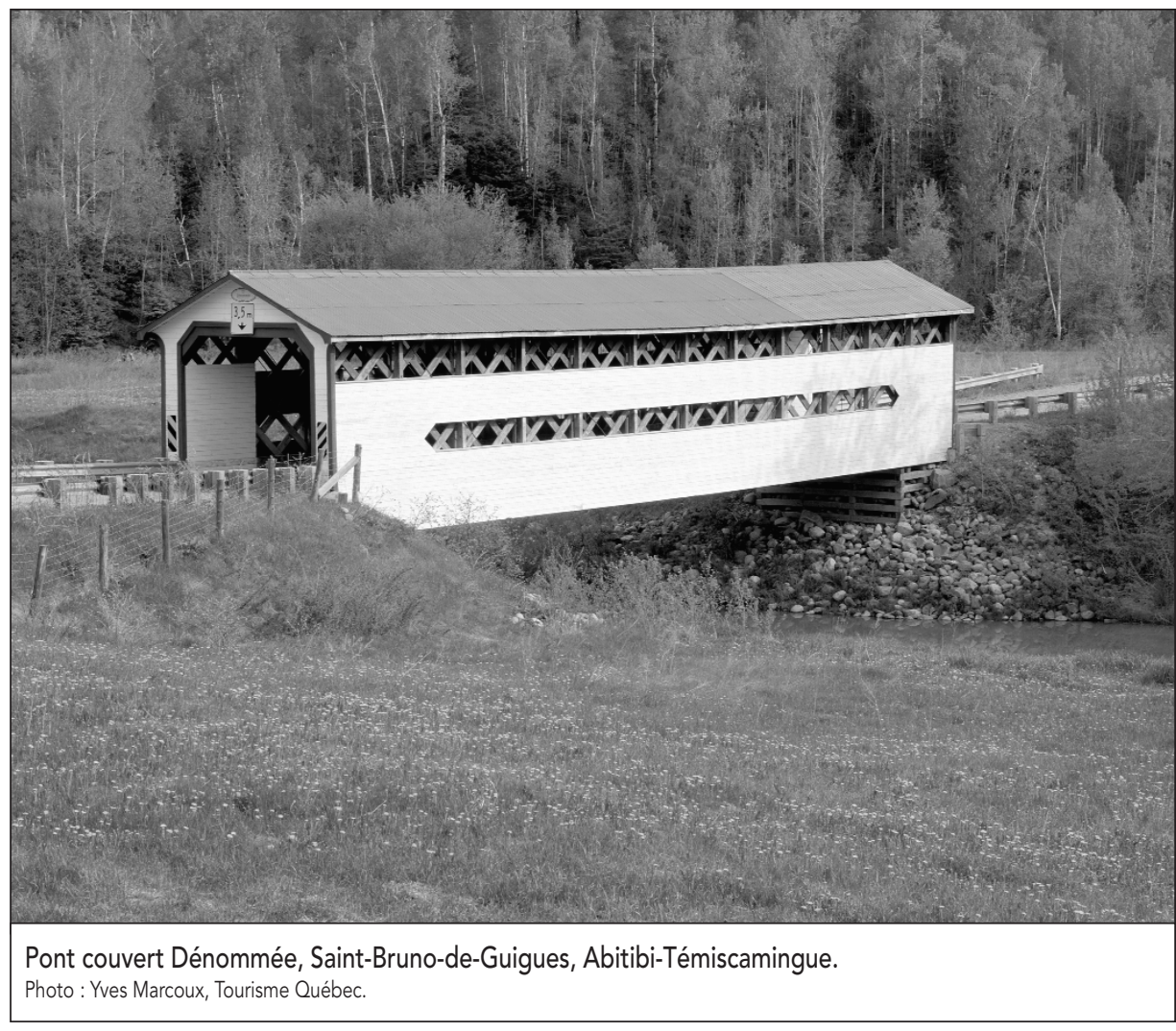

que chacun effectue en cours de voyage seraient des composantes du produit final. En économie, on parle d'un bien composite (Caccomo et Solonandrasana, 2006).

En raison de la complexité du produit, les producteurs (fournisseurs) de chacune des composantes ne perçoivent que vaguement le rôle que leurs biens et services jouent dans l'élaboration du produit final. Par exemple, un repas au restaurant peut jouer un rôle important dans un produit de type "tourisme romantique ", alors qu'il peut tout aussi bien n'avoir qu'une fonction utilitaire (se nourrir) dans d'autres cas (tourisme familial et circuit d'autocar par exemple). Le seul à connaître le rôle de ce repas dans l'élaboration du produit est l'artisan du voyage, soit le touriste lui-même. Les mesures de flux et de dépenses sont donc insuffisantes pour bien saisir le phénomène.

Le touriste aura, consciemment ou non, une façon prédominante de qualifier le voyage qu'il compte entreprendre et c'est cette manière de le nommer, de le penser ou de le sentir qui sera le produit touristique $^{1}$. Ce produit touristique, en particulier le produit anticipé avant le départ, ne peut pas être qu'une somme de composantes assemblées purement au hasard. Le producteur cherchera à mettre en séquence des consommations (des expériences) qui lui permettront de s'approcher de l'idéal recherché. Le touriste élaborera cette séquence dans le but d'assouvir ses besoins et ses désirs.

Par ailleurs, Caccomo et Solonandrasana (2006: 32) font référence aux travaux de Nelson qui précise que le produit touristique constitue un bien d'expérience, c'està-dire, «un bien dont les caractéristiques sont apprises par les consommateurs seulement après leur achat ».

Le touriste est l'artisan du produit qu'il consommera (Smith, 1994). Le touriste a effectivement la liberté de choisir les structures d'accueil, le mode de transport, les activités et la destination. Les voyagistes et les agences de voyages peuvent participer à l'élaboration de ce produit, mais ce dernier sera beaucoup plus qu'un billet d'avion, un hôtel et la location d'une automobile. Même les forfaits «tout compris" comptent une part d'imprévus où le touriste sera appelé à faire certains choix qui le conduiront à vivre une expérience unique. 
Bref, le produit touristique est confectionné par le touriste lui-même ; il se compose d'un assemblage d'éléments disparates, dont certains ne peuvent être prévus à l'avance, et le touriste n'aura une idée complète et juste du produit qu'une fois qu'il aura fini de le consommer.

\section{Les attractions touristiques}

Le terme attraction en tourisme semble à peine mieux cerné que le terme produit et les deux sont parfois employés indistinctement. On s'entend généralement pour dire que l'attraction est ce qui attire le touriste.

Pour Lew (1987), une attraction touristique est composée de tous les éléments localisés ailleurs qui attirent le voyageur hors de son lieu de résidence ${ }^{2}$. Dans un article intitulé «A Framework of Tourist Attraction Research », celui-ci recense et classifie plus d'une cinquantaine d'études et de modèles qui traitent des attractions.

Partant de la définition du paragraphe précédent, l'article regroupe des études très disparates dans lesquelles les attractions peuvent inclurent des paysages, des activités et des expériences. II précise qu'il est parfois difficile de distinguer entre ce qui est une attraction et ce qui n'en est pas une. La catégorisation qu'il tente de faire de tous ces objets est louable et nécessaire, mais elle aboutit en une nomenclature peu opérationnelle, car les objets sont trop disparates. C'est aussi l'avis de Leiper (1990), qui, dans un article subséquent, critique la catégorisation proposée par Lew. II mentionne aussi que le terme attraction est largement utilisé dans la littérature, mais que le traitement qui en est fait manque de cohésion et de rigueur. Nous croyons personnellement que les attractions, telles que répertoriées par Lew, cachent en réalité les trois concepts décrits dans le présent article, soit le produit, l'attraction et l'attrait.

Selon certains classiques (principalement MacCannell, 1979 et Leiper, 1990), une attraction serait tout élément, tangible ou intangible, qui, à partir d'une mise en valeur appropriée de ressources naturelles ou culturelles, aurait la capacité d'attirer un touriste. En général on s'entend pour dire que l'attraction est composée de trois éléments : un objet (un noyau ou un événement), un marqueur (un acte de promotion ou de mise en valeur dudit objet) et un touriste (susceptible d'être attiré par l'objet en question).
Ce lien entre un objet, un touriste et une promotion ne se fait pas au hasard. II y a nécessairement un promoteur. Dans la majorité des cas, ce promoteur est un entrepreneur touristique. Ainsi, pour nous, l'attraction est d'abord et avant tout une entreprise qui contribue à l'offre touristique. Avec le transport et les structures d'accueil, les attractions forment le système de l'offre touristique (voir plus loin le Modèle touristique régional - MTR à ce sujet).

\section{Produits touristiques vs attractions touristiques}

II y a donc une différence importante entre un produit et une attraction touristique. Caccomo et Solonandrasana (2006) nous aident à y voir plus clair en distinguant entre le bien découverte et le bien d'évasion.

Dans le bien découverte, le touriste est spectateur. Le meilleur exemple de ce type de bien est le musée. Le touriste se déplace à l'intérieur du musée et regarde, en spectateur, ce qui y a été mis à son intention. Quand l'expérience de la visite d'un musée est placée sur des axes de temps (en $X$ ) et de satisfaction (en Y), on observe une hausse rapide du niveau de satisfaction dès que le touriste entre dans l'établissement. Une fois la visite terminée, ou à partir du moment où le visiteur sent qu'il en a assez vu (généralement après quelques heures), celui-ci quitte le musée et le niveau de satisfaction redescend. Pour retrouver un niveau de satisfaction élevé, le touriste devra passer au prochain «bien découverte».

Sur ces mêmes axes de temps et de satisfaction, le bien d'évasion se comporte différemment. Le haut niveau de satisfaction peut perdurer très longtemps. Prenons l'exemple du tourisme balnéaire. À partir du moment où le visiteur atteint sa destination $^{3}$, le niveau de satisfaction monte rapidement et ne redescend qu'au moment où le touriste réintègre son "monde ordinaire ${ }^{4}$ ». Dans le cas du bien découverte, la satisfaction est en lien avec un équipement (dans notre exemple il s'agit d'un musée), alors que dans le cas du bien d'évasion, la satisfaction est en lien avec un état d'esprit (dans notre exemple, vivre l'expérience du tourisme balnéaire). Cette distinction correspond à celle que nous faisons entre une attraction (bien découverte) et un produit touristique (bien d'évasion).

\section{Les attraits}

L'attrait, quant à lui, réfère à une notion plus large que l'attraction. L'attrait n'existe pas spécifiquement pour le touriste. Par exemple, un paysage ou une production culturelle locale sont des éléments qui peuvent rendre une ville ou une région attrayante sans pour autant que ces attraits existent à des fins spécifiquement touristiques. Marc Laplante (1983) a déjà suggéré qu'il serait peut-être souhaitable de distinguer entre ce qui existe spécifiquement pour le touriste (une attraction ${ }^{5}$ ) et ce qui existe indépendamment de ce dernier (un attrait). Nous croyons effectivement que cette distinction est nécessaire.

Les attraits sont les ressources naturelles et culturelles, présentes sur le territoire, qui ont le potentiel ${ }^{6}$ de contribuer au produit touristique. Ces attraits peuvent servir de toile de fond lors d'un voyage (paysages, coutumes locales, etc.) ou avoir été mis en valeur par des entrepreneurs pour les rendre accessibles aux touristes. À ce moment-là, les attraits servent de base aux attractions.

Alors que les ressources et le patrimoine semblent pouvoir être utilisés comme synonymes pour décrire ce qu'est un attrait, les économistes apportent quelques nuances. "Le patrimoine désigne un groupe de biens pris dans une relation de transmission. II est ce qui vient des pères et porte implicitement l'obligation de transmettre aux enfants » (Barthélemy et al., 2006: 109).

L'intérêt de référer au patrimoine plutôt qu'aux ressources pour définir l'attrait est cette idée de transmission aux générations futures. C'est tout à fait dans l'esprit du développement durable et c'est moins porteur de connotations négatives qu'une référence directe aux ressources. Ces dernières sont en effet plus facilement associées à une exploitation sans retenue du patrimoine collectif.

Mais il y a aussi des inconvénients à utiliser le terme patrimoine. La mise en place d'un développement durable de la ressource est davantage une aventure systémique que l'attribut d'une ressource. De plus, «le terme de patrimoine ne va cesser de renvoyer à l'expression de deux logiques radicalement opposées : celle d'intérêts individuels marchands et celle de collectifs qui cherchent à se définir à travers un patrimoine commun » (idem : 110). 
Dans le but d'éviter toute ambiguïté, nous avons opté pour l'utilisation du terme plus neutre de "ressources ", tout en gardant à l'esprit toutefois que ces ressources doivent faire l'objet d'une exploitation responsable et lucide. Ainsi, nous employons les expressions "ressources naturelles " et "ressources culturelles » pour définir ce qu'est un attrait ${ }^{7}$.

Produits, attractions et attraits

En mathématique, le produit est la résultante de la multiplication de plusieurs nombres. Dans le modèle que nous proposons, le produit serait la résultante de la juxtaposition de chacune des expériences vécues lors d'un déplacement, amplifiée par le décor nature et culture dans lequel se déroulent ces ex- périences. Les attraits forment le cadre dans lequel prennent place l'offre et la matière première des attractions. Le cadre donnera de l'authenticité au produit, dans la mesure où le produit n'aura pas été parachuté à cet endroit par pur hasard.

Si nous voulions mettre ces composantes sous forme d'équation, nous pourrions dire que le produit $(P)$ est fonction des composantes de l'offre $(O)$ et des attraits $(A)$ : $\mathrm{P}=\mathrm{P}((\mathrm{O} 1, \mathrm{O} 2, \mathrm{O} 3, \ldots, \mathrm{Oi})$; (A1, A2, $\mathrm{A} 3, \ldots$, Ak)).

Voici un exemple relatif au tourisme balnéaire :

Le produit $(P)$

P. Le tourisme balnéaire
L'offre $(\mathrm{O})^{8}$

01: Hôtel en bord de mer

O2: Restaurants de fruit de mer

O3: Activités organisées sur la plage

Oj : ...

Les attraits $(A)$

A1: La mer

A2 : Un climat tropical

A3 : Une culture locale tournée vers la mer Ak: ...

Le nombre (i) et la qualité des expériences vécues lors du voyage ainsi que le nombre (k) et la qualité des attraits contribueront à la cohérence du produit touristique imaginé au départ par le touriste. L'équation pourrait aussi compter des éléments négatifs (-Ox ou -Ay) qui contribueraient à ré-

Figure 1

Adaptation du modèle touristique régional

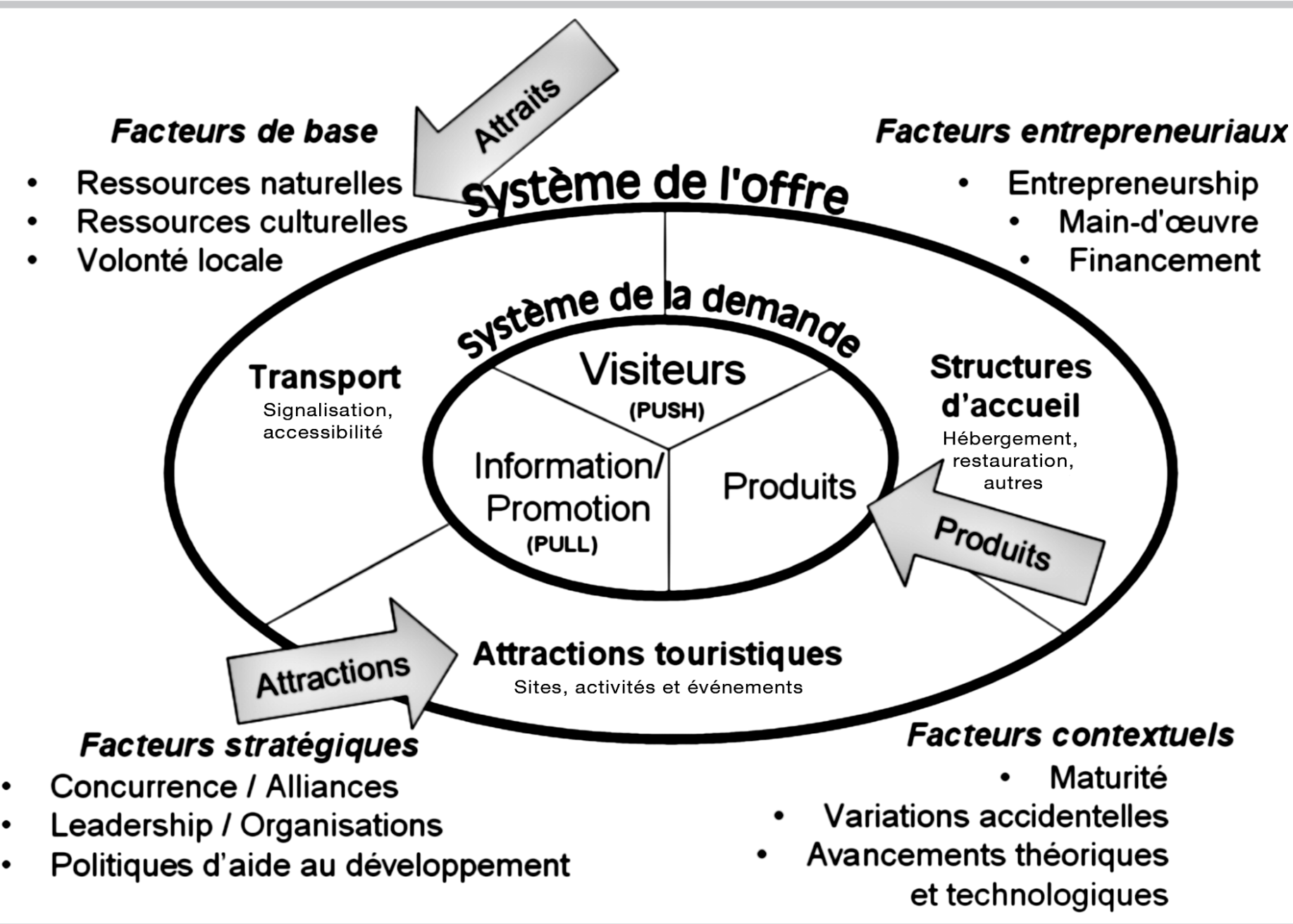


duire la qualité du produit. Dans notre exemple de tourisme balnéaire, la pollution, la violence dans les rues, des hôtels hors de prix qui n'attirent pas les jeunes (associés à l'image du tourisme balnéaire), etc. contribueraient à réduire la cohérence et la qualité du produit.

II serait réducteur de la complexité du phénomène touristique de dire que l'équation $\mathrm{P}=\mathrm{P}((\mathrm{O} 1, \mathrm{O} 2, \mathrm{O} 3, \ldots, \mathrm{Ok}) ;(\mathrm{A} 1, \mathrm{~A} 2$, $A 3, \ldots, A l))$ suffit à résumer l'activité touristique. Dans mes travaux de thèse, je propose un modèle pour expliquer le fonctionnement du tourisme en région (de Grandpré, 2005). Ce modèle (le MTR) donne aux produits touristiques, aux attractions et aux attraits des fonctions spécifiques et complémentaires de la mise en tourisme régionale.

\section{Le modèle touristique régional (MTR)}

Le but du présent article n'est pas de présenter ce modèle ${ }^{9}$, mais seulement d'illustrer la place qu'occupent les produits, les attractions et les attraits dans un contexte touristique régional.

Au centre du modèle se trouve le système de la demande. À sa plus simple expression, ce système est la résultante de l'interaction entre les trois éléments suivants : le besoin qu'éprouve une personne de quitter son lieu habituel de vie pour un certain temps (push); la promotion / information à sa disposition (pull); et les produits touristiques qui sont à sa portée. Derrière ce système de la demande se cache tout l'arsenal des outils marketing (notoriété, image, processus de prise de décision, marchéage, intermédiaires du voyage, etc.).

Vient ensuite le système de l'offre qui, à sa plus simple expression encore une fois ${ }^{10}$, se résume à trois composantes: le transport, les structures d'accueil et les attractions. II s'agit d'entreprises qui offrent directement des services aux touristes. Le système de l'offre est la partie la plus apparente pour les voyageurs, c'est aussi l'endroit où les dépenses sont réalisées (mais pas exclusivement ${ }^{11}$ ).

Enfin, en périphérie du système, les facteurs extérieurs sont formés de l'ensemble des éléments susceptibles d'aider ou de nuire au positionnement touristique d'une région. Hormis les attraits (facteurs de

base), ces éléments extérieurs ne sont généralement pas directement perceptibles par le voyageur. Ils n'en influencent pas moins la qualité du produit que ce dernier consommera. Les facteurs extérieurs comptent quatre groupes: les facteurs de base, qui sont les fondements de l'identité d'un territoire; les facteurs stratégiques, sous le contrôle des acteurs locaux et régionaux; les facteurs entrepreneuriaux liés au bon fonctionnement des entreprises; et les facteurs contextuels qui évoluent indépendamment de la volonté des acteurs.

Comme la figure 1 l'illustre (flèches), le produit touristique, les attractions et les attraits ont chacun un rôle particulier à jouer dans la dynamique touristique régionale. À titre d'exemple, un voyage de ski est un produit, une montagne un attrait et un centre de ski une attraction.

Sur cette base, nous pourrions donc définir le concept de produit de la manière suivante: le produit touristique est d'abord et avant tout la représentation mentale qu'un individu se fait du voyage qu'il désire entreprendre. Celle-ci est formée d'expériences culturelles et de loisirs qu'il cherchera à vivre lors de son voyage. Cette pulsion lui vient d'un besoin à satisfaire et s'actualisera sous l'influence de l'information dont il dispose, de la promotion dont il est la cible, ainsi que des pos-

\section{Tableau 1}

\section{Exemples de produits touristiques}

Thématique

Agrotourisme

Écotourisme

Tourisme autochtone

Tourisme blanc

Tourisme bleu

Tourisme culinaire

Tourisme culturel

Tourisme d'affaire / congrès

Tourisme d'apprentissage

Tourisme d'aventure / Grande aventure

Tourisme familial

Tourisme de santé

Tourisme gai

Tourisme religieux

Tourisme social

Tourisme urbain

Tourisme vert

Etc. sibilités qui s'offrent à lui (palette de choix de destinations potentielles, temps et argent disponibles, pression sociale, etc.).

Ces produits peuvent prendre trois formes: une expérience thématique, une destination ou une activité. Dans les trois cas, il s'agit de vivre une série d'expériences qui contribueront à façonner le produit. Considérant la multitude d'expériences potentielles lors d'un voyage, chaque déplacement est unique. II y a donc autant de produits qu'il y a de voyages. Heureusement, il est possible de regrouper ces produits à l'intérieur de grandes catégories. Le tableau 1 présente des exemples de produits.

Dans le contexte du MTR, l'attraction est, quant à elle, une des trois composantes de l'offre. C'est une entreprise qui met en valeur un attrait à des fins touristiques. L'attrait naturel (plan d'eau, montagne, climat, etc.) ou culturel (fait historique, production culturelle, art, etc.) est ainsi organisé, transformé et publicisé dans le but explicite d'intéresser des touristes. Des exemples d'attractions sont: les parcs à thèmes, les institutions muséales, les festivals, les casinos, les terrains de golf ${ }^{13}$, les croisières d'observation des baleines, des visites guidées, etc. 


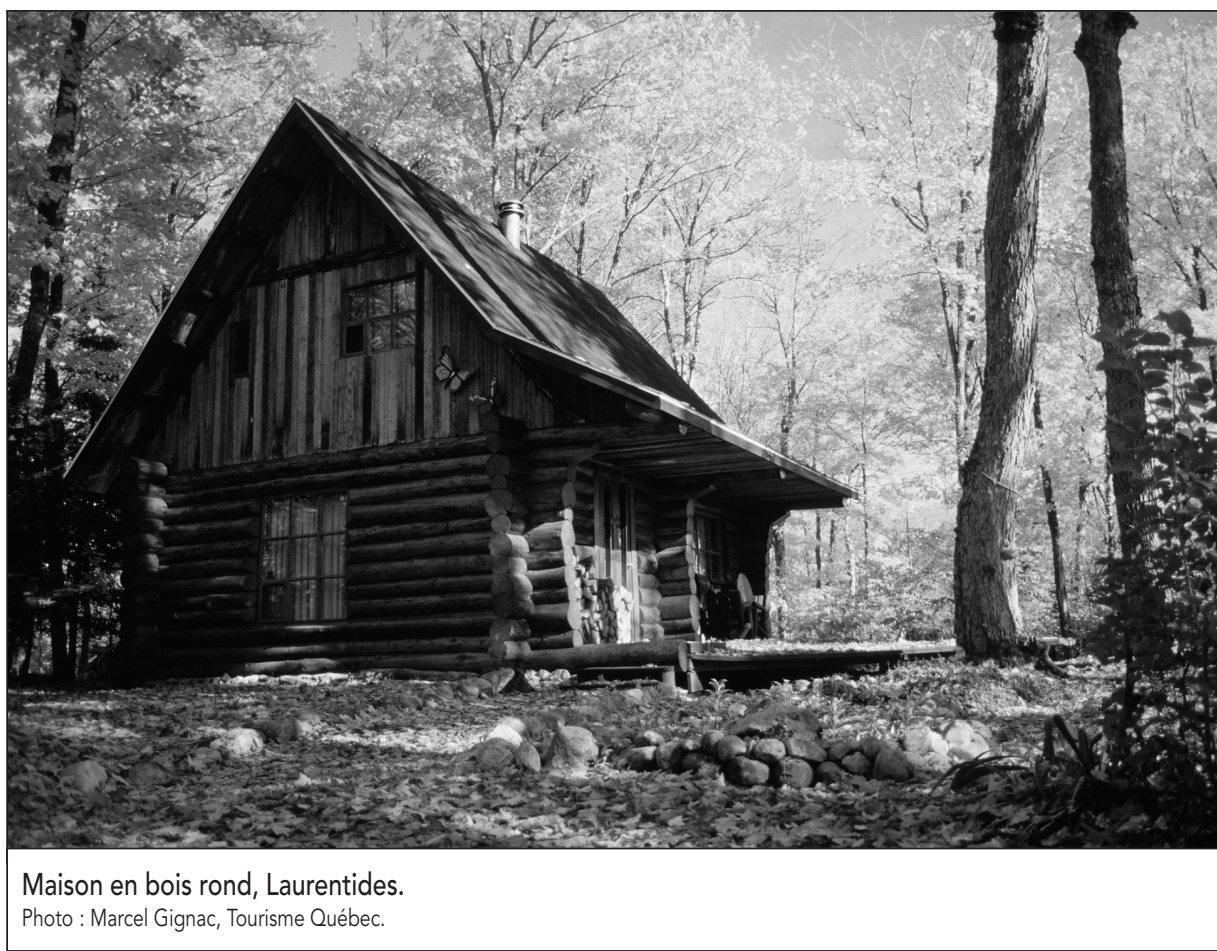

Enfin, l'attrait est une ressource naturelle ou une ressource culturelle qui a le potentiel de rendre un territoire attrayant pour un touriste. Cette ressource, ou partie de la ressource, peut avoir été transformée en attraction à l'intention du touriste ou non. Des exemples seraient des montagnes, des paysages, le climat, des coutumes locales et même l'attitude des résidants d'un territoire. Ainsi, la "joie de vivre " des Québécois peut être considérée comme un attrait et éventuellement devenir un produit ${ }^{14}$.

La démarcation entre produits, attractions et attraits dans le contexte du MTR est claire. Ce qui n'empêche pas qu'un objet puisse être les deux, voire les trois à la fois. L'attraction est presque toujours un attrait au départ, mais un attrait n'est pas nécessairement une attraction. Un attrait peut être un produit. Les attraits suivants sont des produits dans la mesure où un touriste, quelque part, imagine une séquence de consommations qui lui fera vivre l'expérience de l'attrait: les paysages de la Provence, le climat des Antilles, l'ambiance de Charlevoix, les plaines d'Abraham, le débarquement en Normandie. Si la destination est suffisamment développée, le touriste trouvera sur place des attractions qui l'aideront à vivre l'expérience convoitée.
Toujours dans le contexte du MTR, il est possible qu'une des composantes du système de l'offre (structure d'accueil et transport) soit à la fois attraction et produit. Nous pensons par exemple à l'Orient Express, au château Frontenac, aux restaurants très hauts cotés dans les guides touristiques et à tout autre équipement d'accueil et de transport qui offrent des expériences exceptionnelles aux voyageurs, des expériences qui valent le voyage.

\section{Conclusion}

Les concepts de produits, d'attractions et d'attraits peuvent difficilement être expliqués en dehors du contexte dans lequel ils sont utilisés. Que ce soit dans un contexte de marketing, de planification, industriel, de géographie, d'économie ou, comme c'est le cas ici, touristique, ces notions peuvent prendre des significations différentes. Nous appuyant sur le MTR et quelques ouvrages spécifiques à ce sujet, nous avons tenté dans cet article de réduire un peu la confusion qui semble régner autour de ces termes.

La définition des concepts, tels qu'ils sont présentés ici, n'est pas sans implications pour l'industrie touristique. La dimension intangible du tourisme, le rôle des biens publics, les désirs parfois excentriques des touristes, les phénomènes de mode, les défis d'adapter l'offre à la demande, sont autant d'enjeux que soulèvent les concepts décrits dans le présent article.

Ce qui peut paraître paradoxal, c'est qu'une approche par produit signifie qu'il faille d'abord penser la demande avant de penser l'offre. En effet, le produit est d'abord et avant tout dans la tête du touriste et non sur le terrain. Au Québec, les associations touristiques régionales (ATR), les offices de tourisme, les chambres de commerce et tout autre organisme de promotion et de développement touristique local et régional sont limités dans leur capacité à mettre en place une véritable stratégie de positionnement par produit à cause justement de cette perspective (celle de l'offre ${ }^{15}$ ) qui vient de leur mission et de leur mandat.

L'autre difficulté vient de la délimitation du territoire que les organismes de promotion et de développement touristiques ont la charge de mettre en valeur. Le touriste ne fait pas de distinction entre une région administrative et une autre au moment où il élabore son produit. II n'hésitera pas à franchir la frontière qui sépare ces régions s'il sent que c'est au bénéfice du produit qu'il est en train de construire. Certaines régions l'ont compris et n'hésitent pas à faire fi de ces fausses frontières (fausses du point de vue touristique). La municipalité de SaintDonat par exemple, située dans Lanaudière, perçoit bien que son offre correspond davantage à l'image que le touriste se fait des Laurentides. Elle n'hésite donc pas à s'identifier à cette région voisine dans certaines promotions touristiques. Le Québec maritime est un autre exemple. Les territoires membres proviennent de plusieurs régions touristiques; ce qui les unit c'est la volonté de certains touristes de vivre une expérience de bord de mer particulière. De la même manière, les «routes touristiques " et les sentiers au Québec sont généralement plus intéressants s'ils ne se confinent pas à une seule région. Nous croyons que des produits touristiques restent inexplorés ou sous-développés, au Québec, à cause justement du découpage (de Grandpré, 2000). 
Il est vrai que les organismes de développement local et régional sont liés à leur mission de mettre en valeur un territoire prédéterminé. Peut-être qu'en tourisme serait-il plus rentable pour eux, et pour le Québec en général, de chercher des alliances en dehors de leur territoire? À moins que la solution ne soit ailleurs. Peut-être serait-il intéressant pour le Québec de songer à fonder des ATP (associations touristiques de produits)?

\section{François de Grandpré est professeur au} département d'études en loisir, culture et tourisme à I'Université du Québec à Trois-Rivières.

\section{Notes}

1 On peut penser un voyage qui compterait deux ou trois produits. Le cas par exemple d'un touriste en voyage d'affaires qui prévoit aussi faire un voyage d'agrément. Dans ce cas, cette même personne aura deux identités différentes lors du même voyage, mais sans doute pas en même temps.

2 "A tourist attraction consists of all those elements of 'non-home' places that draw discretionary travelers away from their homes. " (Lew, 1987, p. 54)

3 On peut argumenter que cette satisfaction peut commencer avant l'arrivée à la destination (anticipation) et se poursuivre après le retour (souvenirs).

4 En référence au système du touriste de Jafar Jafari (1988).

5 "Attraction ", "attraction touristique " et "attrait touristique» seraient synonymes. Le mot "attrait » utilisé seul référerait aux ressources naturelles et culturelles qui ne sont pas nécessairement en lien avec le tourisme. On peut aussi dire « attrait naturel » et « attrait culturel».

6 Pour être une ressource touristique, l'attrait doit servir directement ou indirectement dans l'élaboration du produit touristique, sinon ce n'est pas une ressource. À l'étape de la planification régionale, l'attrait doit avoir le potentiel de servir un touriste dans l'élaboration ou la consommation de son produit pour être considérée une ressource.
7 L'équivalent anglais du mot « attrait » n'est pas évident. II est traduit par attraction ou appeal dans le dictionnaire anglais-français / français-anglais Harrap's Shorter (1998). Compte tenu du sens et du contexte dans lesquels nous l'utilisons, nous proposons plutôt l'emploi du terme attributes.

8 L'offre est constituée de trois catégories d'éléments: le transport, les structures d'accueil et les attractions.

9 Le modèle qui est présenté à la figure 1 est expliqué en détail dans un article soumis à la revue Tourisme \& Territoires / Territories \& Tourism (de Grandpré, à paraître).

10 Les modèles sont généralement des simplifications de la réalité.

11 || y a des exceptions: les intermédiaires du voyage et les institutions financières par exemple.

12 La liste peut paraître compter des éléments non mutuellement exclusifs, comme ici pour le camping qui est à la fois une activité et un mode d'hébergement. En fait, il faut questionner le voyageur pour savoir si son but premier est de faire du camping ou autre chose. Faire du plein air, découvrir une région à bas prix peuvent conduire à faire du camping; le camping devient alors un moyen et non une fin.

13 Des équipements, comme certains terrains de golf, ne reçoivent parfois qu'une infime partie de touristes. La clientèle peut se limiter à une clientèle de proximité. II est difficile de fixer la proportion de touristes qu'un équipement doit recevoir pour être qualifié de "touristique». Dans le cas des festivals, le chiffre de $15 \%$ est généralement utilisé pour distinguer entre un festival qui est touristique et un autre qui ne l'est pas.

14 Et ce, même si le Québécois n'était pas plus accueillant qu'un autre peuple. L'important pour "devenir un produit» est que le touriste pense qu'il s'agit d'un produit et qu'il veuille en faire l'expérience lors d'un voyage.

15 A fortiori quand l'organisme représente ses membres davantage que son territoire.

\section{Bibliographie}

Barthélemy, Denis, Martino Neiddu, et FrankDominique Vivien (2006), «Refondation critique de la notion de patrimoine ", La Pensée, n 347, juillet-septembre.

Caccomo, Jean-Louis, et Bernardin Solonandrasana (2006) [2 ${ }^{\mathrm{e}}$ éd.], L'innovation dans l'industrie touristique - Enjeux et stratégies, L'Harmattan, Paris.

de Grandpré, François (2000), "Le découpage des régions touristiques du Québec - Éléments d'analyse et pistes de recherches ", Téoros, vol. 19, n³, p. 40-43.

de Grandpré, François (2005), Proposition d'une modélisation du système touristique régional (MTR) et application au Québec, thèse de doctorat, Université Paul-Cézanne-AixMarseille.

de Grandpré, François (à paraître), « Modélisation du tourisme régional », Tourisme et Territoires / Territories \& Tourism, [tourter.com].

Jafari, Jafar (1988), "Le système du touriste: Modèles socio-culturels en vue d'applications théoriques et pratiques ", Loisir \& Société / Society \& Leisure, vol. 11, n 1 , p. 59-80.

Jafari, Jafar (2000), Encyclopedia of Tourism, Routledge, Grande-Bretagne.

Kotler, P., P. Filiatrault, et R.E. Turner (2000) [ $2^{e}$ éd.], Le management du marketing, Gaëtan Morin Éditeur.

Laplante, Marc (1983), «Les attractions touristiques: un système à décoder ", Téoros, vol. 2, n 2, p. 14-19.

Leiper, Neil (1990), "Tourist Attraction Systems », Annals of Tourism Research, vol. 17, p. 390-407.

Lew, Alan E. (1987), «A Framework of Tourist Attraction Research", Annals of Tourism Research, vol. 14, n 4, p. 553-575.

MacCannell, Dean (1979), The Tourist - A New Theory of the Leisure Class, New York, Schocken Books.

Smith, S.L.J. (1994) "The Tourism Product", Annals of Tourism Research, n०21, p. 582-595.

Smith, S.L.J. (2003). «A Vision for the Canadian Tourism Industry ", Tourism Management, vol. 24, n², p. 123-133. 